\title{
Reinfection in a healthcare worker with COVID-19 in a hospital in North India
}

\author{
Nazia Nazir, Arun Ahirwar, Shruti Jain \\ Affiliation: Gautam Buddha University, Greater Noida, UP-201310, India. \\ Correspondence: Dr Nazia Nazir; E-mail: nazunazir@gmail.com; Phone: +91 9560102957
}

Citation: Nazir N, Ahirwar A, Jain S. Reinfection in a healthcare worker with COVID-19 in a hospital in North India. Anaesth. pain intensive care 2020;24(5):

The world is in grip of the worst pandemic ever known to mankind in modern times and despite all measures to control, it continues to spread worldwide.

Here, we report a case of reinfection after a gap of 97 days in our hospital. A 26-year-old male was working as a healthcare worker (HCW) in the COVID Intensive Care Unit. After completing his posting of 14 days (active quarantine), he tested positive with SARS CoV-2 by real-time PCR (RTPCR) assay on 3 May 2020 during routine testing, which is done for all HCWs at the end of the active quarantine period as per the hospital policy. He was asymptomatic and his investigations were in the normal range. $\mathrm{He}$ was hospitalized in the isolation ward the next day. He received treatment with tab hydroxychloroquine $(400 \mathrm{mg} \mathrm{BD}$ on the first day followed by $200 \mathrm{mg} \mathrm{BD}$ for the next 6 days), tab oseltamivir $150 \mathrm{mg}$ BD for 7 days, tab Montair LC ${ }^{\mathrm{TM}}$ (a combination of two medicines montelukast and levocetirizine) OD (for 10 days), tab ranitidine $150 \mathrm{mg}$ OD (for 10 days), vitamin $\mathrm{B}$ complex, vitamin $\mathrm{C}$ and zinc. The patient had an uneventful course in the hospital. The two nasopharyngeal swabs (NPS) collected on $10^{\text {th }}$ and $13^{\text {th }}$ May were both negative the infection. He was discharged on $14^{\text {th }}$ and was encouraged to follow home quarantine for the next 14 days.

He re-joined the duties on $30^{\text {th }}$ May and was posted in the non-COVID zone of the hospital. After a gap of 38 days, he was posted in COVID ICU on $7^{\text {th }}$ August 2020. On completion of his posting and 14 days active quarantine, he was sampled by NPS and assay by RT-PCR on 21st August. The test results came out to be positive again. This time too, he remained asymptomatic. He was admitted to the isolation ward and received the same treatment. The patient never required oxygen supplementation.

Preliminary evidence suggests that antibody responses occur in those who have been infected with SARS CoV-2 infection. ${ }^{1}$ Second infection in these patients suggests that sufficient antibody was not generated at the time of the first infection or if they developed, it may not have lasted long enough to prevent re-infection. Reinfection can also happen if there is a very short-lived antibody response without any cellular immunity. Although, in our case, it cannot be confirmed as the antibody titre of the patient during the first episode was not done due to the non-availability of the test.

Ye et al. reported a $9 \%$ proportion of reactivation in COVID-19 patients after discharge from the hospital. Three key risk factors are involved in reactivation; (1) host status, (2) virologic factors, and type and degree of immunosuppression. Host factors include sex, older age, and severity of the disease. The virologic factors associated with an increased risk of reactivation include high baseline viral load and variable genotype. Steroids used as immunosuppressive agents may result in broad immune dysfunctions and potential SARS-CoV-2 reactivation. $^{2}$

In our case, the patient was a young male, asymptomatic, and did not require immunosuppressive therapy. The viral genome was not not available in our institution. However, our patient received antiviral therapy (oseltamivir). It has been suggested that SARS-CoV-2 reactivation may occur whenever the antiviral therapy was used. ${ }^{2}$

The reinfection of a healthy individual in a short span of fewer than 100 days has several implications in terms of herd immunity, vaccination schedules, 
and antibody titres in the convalescent serum and prevalence of infection in population. We also need to study the life span of antibodies.

In conclusion, this case highlights the importance of vaccination in individuals with the previous infection. People with the previous infection cannot be complacent in following social distancing and wearing of masks.

\section{REFERENCES}

1. Long $Q$, Liu B, Deng $H$, Wu GC, Deng K, Chen YK, et al. Antibody responses to SARS-CoV-2 in patients with COVID-19. Nat Med. 2020. [PubMed] DOI: 10.1038/s41591-020-0897-1

2. Ye G, Pan Z, Pan Y, Deng Q, Chen L, Li J, et al. Clinical characteristics of severe acute respiratory syndrome coronavirus 2 reactivation. J Infect. 2020 May;80(5):e14-e17. [PubMed] DOI: 10.1016/j.jinf.2020.03.001

\section{'My Most Memorable Patient'}

\section{An ophthalmologist can save lives too; by keeping an open mind}

\section{Jan E. Siegersma}

The Netherlands; E-mail: eizesmail@hotmail.com

Taking my residency into account I have now been in the field of ophthalmology for 20 years. That is a good moment for some reflection. Have I chosen the right specialty? Have I seen some remarkable patients? Not a lot of medical students starting medical school desire to become an ophthalmologist. In fact amongst most medical students ophthalmology is not quite popular. It is not 'sexy' or 'heroic' like surgery, traumatology or intensive care medicine. It is considered by many as boring. In ophthalmology you do not save lives. The only thing you do is prescribing glasses, ... is it not? Most of us doctors have started their careers with the desire to save lives. To be there where the action is: in the OR or ER. But being an ophthalmologist you can also save lives. I wondered how many lives I had saved. At least a few. This is one of them. This is the story of 'My Most Memorable Patient'.

The story is the well-known story about 'Everybody', 'Somebody', 'Anybody' and 'Nobody'. I am sure you must have heard about that story. ${ }^{1}$

It was during my residency at an eye department of a University Medical Centre somewhere in the Netherlands. The workload was very high, maybe well too high. The story is about a patient with Marfan syndrome (MFS). For those of us who are not well familiar with MFS, it is a connective tissue disorder. It can give a range of cardiovascular, ocular, neurological, musculoskeletal and neurological disorders. ${ }^{2}$ The cardiovascular abnormalities include thoracic aortic aneurysm, aortic dissection and mitral valve prolapse, which can be lifethreatening. Ocular abnormalities include tendency to retinal detachment, high myopia, lens subluxation, early onset glaucoma and early cataract.

Because of the possible life-threatening complications it is important that these patients are under regular control by a multidisciplinary team. In our hospital these patients were seen every year by a cardiologist, ophthalmologist, neurologist and also a dermatologist. They got an ultrasound exam to detect aortic aneurysm/dissection. People made a kind of carousel visiting all the different involved specialisms on one day. They used to show up with a bunch of paperwork to be filled out by every doctor they were consulting that day.

Someone with MFS came to me during my residency for a periodic eye check-up. I had never seen him before. He had a long and complicated ophthalmic medical history. He had been a patient at our department for many years. In all those years he had been seen by almost all our doctors, including many residents, all the vitreoretinal surgeons and even our head of the department. He had had all the eye complications related to MFS you could imagine. He had had several retinal detachment surgeries in both of his eyes as well as lens replacement surgery for the for MFS typical lens subluxation. I was surprised by the fact that the patient had not given me the paperwork to be filled out for the MFS screening program. So I asked him, if he was in the screening program. He was not! He had never heard of it. I was astonished. In all those years he had been patient with us, 
nobody had referred him to the screening program. And nobody had ever asked if he was in the program. I contacted the coordinator of the program and referred the patient immediately. Of course I talked about this case in our weekly department meeting. One year later the same patient came again to me for his yearly eye exam. He was very grateful for having saved his live. Soon after my referral to the MFS screening program he had been called in. There was found a large thoracic aortic aneurysm, and aortic prosthetic surgery was needed. Also all his family members had been called in for screening.

What can we learn from this case?

MFS patients have a high risk of developing an aortic aneurysm or aortic dissection. This is life threatening. Everyone with MFS should, therefore, be in a screening program. It is incredible that someone with MFS could have been a patient on an eye department of a University Hospital for many years, without having been referred to the MFS screening program in the same hospital. Why has never someone of all those doctors in all those years checked if that patient was participating in the screening program? Was it indolence? Had it to do with the high workload? I think it was, because everyone was assuming that patient would have been in the screening program, because it would have been incredible he was not. The mistake was that no one verified his assumption. We should never assume something without verification! In aviation many serious flight accidents have happened because of something was assumed. Therefore, they work now with checklists. In medicine, lives can be lost by assuming. My advice is to ask questions to confirm your assumption, even if the assumption looks evident or the question looks silly or stupid. We should also work more with checklists, like it is done in modern aviation. Don't focus only on your own specialty, but focus beyond your own domain.

So...every doctor can save lives. Even an ophthalmologist! By keeping an open mind. On the other hand, not having an open mind, or making assumptions can cost lives!

\section{REFERENCES}

1. LOLLY DASKAL. The Story of Everybody, Somebody, Anybody And Nobody (Blog). Available at https://www.lollydaskal.com/leadership/story-everybody-somebody-anybody-nobody/

2. Marfan Syndrome, Medscape. Available at https://emedicine.medscape.com/article/1258926-overview 